University of Zurich

Department of Economics

Working Paper Series

ISSN 1664-7041 (print)

ISSN 1664-705X (online)

Working Paper No. 212

\title{
On the Equivalence of Bayesian and Dominant Strategy Implementation: The Case of Non-Linear Utilities
}

Alexey Kushnir and Shuo Liu

November 2015 


\title{
On the Equivalence of Bayesian AND
} Dominant Strategy Implementation: The Case of Non-Linear Utilities*

\author{
Alexey Kushnir Shuo Liu
}

November 23, 2015

\begin{abstract}
We extend the equivalence between Bayesian and dominant strategy implementation established by Gershkov et al. (Econometrica, 2013) to environments with non-linear utilities satisfying the average single-crossing property and the convex-valued assumption. The new equivalence result produces novel implications to the literature on the principal-agent problem with allocative externalities, environmental mechanism design, and public good provision.
\end{abstract}

Keywords: Bayesian implementation, dominant strategy implementation, mechanism design, non-linear utilities, single-crossing

JEL Classification: D82

\footnotetext{
*Alexey Kushnir: Tepper School of Business, Carnegie Mellon University, Posner Hall 237, Pittsburgh, PA 15213, USA; Shuo Liu: Department of Economics, University of Zürich, Schönberggasse 1, CH-8001, Zürich, Switzerland. We are very grateful to Jean-Michel Benkert, Tilman Börgers, Satoshi Fukuda, Navin Kartik, Alejandro Manelli, Nick Netzer, Philipp Strack, Mehmet Bumin Yenmez, seminar participants at Lucerne (ZWE 2015) and Zürich for discussions and useful suggestions.
} 


\section{Introduction}

For social choice environments with linear utilities and independent, one-dimensional private types, Gershkov et al. (2013) have recently shown a prominent result that for any Bayesian incentive compatible (BIC) mechanism there exists an equivalent dominant-strategy incentive compatible (DIC) mechanism that yields the same interim expected utilities to all agents and generates the same social surplus.$^{1}$ Hence, for a wide range of objectives, including revenue and efficiency maximization, the mechanism designer can restrict himself to more robust DIC mechanisms and gain nothing from designing possibly more complex mechanisms with Bayes-Nash equilibria.

The main contribution of this paper is to extend the BIC-DIC equivalence result to environments with non-linear utilities satisfying two assumptions. First, we assume that each agent's utility has the average single-crossing property that is a natural generalization of the single-crossing property to Bayesian settings. We use this generalization to characterize BIC mechanisms in terms of a monotonicity condition and an envelope formula. To understand the limitations of the average singlecrossing property, we also fully characterize the set of utility functions satisfying this assumption.

Second, we assume that the mapping of all agents' utilities, as a mapping from the set of feasible allocations to the space of possible utilities, is convex-valued. Though this condition might be restrictive in general settings, it is trivially satisfied for linear utilities defined on a simplex (as in Gershkov et al., 2013) and for any symmetric setting. We also illustrate that many important papers in the literature on principal-agent problems, environmental mechanism design and public good provision analyze settings satisfying this assumption.

Assuming the average single-crossing property and the mapping of all agents' utilities being convex-valued, we establish the BIC-DIC equivalence for non-linear environments. In addition, we provide conditions on agents' utilities when for a given BIC mechanism one could find a DIC mechanism that yields the same interim expected utilities to all agents and generates at least as large expected social surplus.

Finally, we illustrate the significance of our results with several important applications, for which the previous works have little bite (e.g. Manelli and Vincent, 2010; Gershkov et al., 2013). We first

\footnotetext{
${ }^{1}$ The equivalence of mechanisms in terms of the same interim expected utilities is first proposed by Manelli and Vincent (2010). They are also first to establish the BIC-DIC equivalence for standard single-unit, private-value auctions. Goeree and Kushnir (2015) provides an alternative proof of the BIC-DIC equivalence using a novel geometric approach to mechanism design. Kushnir (2015) extends the result to environments with correlated types.
} 
consider the principal-agent problem in a procurement context and illustrate that many influential papers satisfy our main assumptions (e.g., Laffont and Martimort, 1997; Mookherjee and Tsumagari, 2004). In the same context, we study settings with allocative externalities, when agents care not only about their own contracts, but also about contracts received by other agents (e.g., Jehiel et al., 1996). If agents face non-decreasing, convex (concave) contracting costs and positive (negative), concave externalities, then for any BIC mechanism one could find a DIC mechanism yielding the same interim expected utilities to all agents and generating at least as large social surplus. We also establish that the above result holds for environmental mechanism design problems (Martimort and Sand-Zantman, 2013, 2015; Baliga and Maskin, 2003), when agents have linear (concave) benefits and concave (linear) costs of pollution reduction. We finally consider the evergreen problem of public good provision, where in addition to incentive compatibility and individual rationality constraints the budget-balance constraint is of huge importance (e.g., Mailath and Postlewaite, 1990; Ledyard and Palfrey, 1999; Hellwig, 2003; Norman, 2004). When agents have concave utilities and the costs of public good provision is convex, we show that for any $\mathrm{BIC}$ mechanism that is ex ante budget balanced there exists an equivalent DIC mechanism that is also ex ante budget balanced.

The paper is organized as follows. Section 2 presents the model. We discuss the single-crossing properties in Section 3. We prove our main equivalence results in Section 4. Section 5 presents applications. Finally, Section 6 concludes. The Appendix contains omitted proofs.

\section{Model}

We consider environments with a finite set of agents $\mathcal{I}=\{1,2, . ., I\}$ and a compact set of available alternatives $A \subset \mathbb{R}^{k}$ for some natural $k$. Agent $i$ 's utility when alternative $a \in A$ is chosen equals $v_{i}\left(a, x_{i}\right)+t_{i}$, where $x_{i}$ is agent $i$ 's type that is independently distributed according to probability distribution $\lambda_{i}$ with one-dimensional support $X_{i} \subset \mathbb{R}, v_{i}: A \times X_{i} \rightarrow \mathbb{R}$ is some continuously differentiable function with $v_{i x}$ being its derivative with respect to $x_{i}$, and $t_{i} \in \mathbb{R}$ is a monetary transfer. We denote $\mathbf{x}=\left(x_{1}, \ldots, x_{I}\right), X=\prod_{i \in \mathcal{I}} X_{i}$, and $\lambda=\prod_{i \in \mathcal{I}} \lambda_{i}$.

Without loss of generality we consider only direct mechanisms $(q, t)$, where $q: X \rightarrow A$ defines an allocation rule and $t=\left\{t_{i}\right\}_{i \in \mathcal{I}}$, with $t_{i}: X \rightarrow \mathbb{R}$ defines monetary transfers to agents. A mechanism $(q, t)$ is Bayesian incentive compatible or BIC (dominant strategy incentive compatible 
or DIC) if truthful reporting by all agents constitutes a Bayes-Nash equilibrium (a dominant strategy equilibrium). We also say that an allocation rule $q$ is BIC (DIC) if there exist a payment rule $t$ such that mechanism $(q, t)$ is Bayesian incentive compatible (dominant strategy incentive compatible).

When all agents report their types truthfully and agent $i$ 's type is $x_{i}$, we write his utility as $u_{i}(\mathbf{x})=v_{i}\left(q(\mathbf{x}), x_{i}\right)+t_{i}(\mathbf{x})$ and his interim expected utility as $U_{i}\left(x_{i}\right)=E_{\mathbf{x}_{-i}}\left(v_{i}\left(q(\mathbf{x}), x_{i}\right)+t_{i}(\mathbf{x})\right)$. The expected social surplus is defined as $E_{\mathbf{x}}\left(\sum_{i \in \mathcal{I}} v_{i}\left(q(\mathbf{x}), x_{i}\right)\right)$ or, equivalently, as the sum of agents' ex ante expected utilities minus the sum of agents' ex ante expected transfers. As in Gershkov et al. (2013), we employ the following notion of equivalence.

Definition 1. Two mechanisms $(q, t)$ and $(\tilde{q}, \tilde{t})$ are equivalent if and only if they yield the same interim expected utilities for all agents and generate the same expected social surplus.

\section{The Single-Crossing Properties}

In this section, we introduce a novel condition that we call the average single-crossing property. We use this property to characterize Bayesian incentive compatible mechanisms in terms of a monotonicity condition and an envelope formula similar to how the single-crossing property is used to characterize dominant strategy incentive compatible mechanisms.

We consider connected type spaces with $X_{i}=\left.\left[\underline{x}_{i}, \bar{x}_{i}\right] \subset \mathbb{R}\right|^{2}$ As a motivation, let us first consider the single-crossing property (see Mookherjee and Reichelstein, 1992).

Definition 2. Function $v_{i}$ satisfies the single-crossing property when for any $a, a^{\prime} \in A$ if there exists type $y$ such that $v_{i x}(a, y)>v_{i x}\left(a^{\prime}, y\right)$, then for any type $x \in X_{i}, v_{i x}(a, x)>v_{i x}\left(a^{\prime}, x\right)$.

If function $v_{i}$ satisfies the single-crossing property for each $i \in \mathcal{I}$ then dominant strategy incentive compatibility can be characterized by a monotone-marginal condition and an envelope formula:

Proposition 1 (Mookherjee And Reichelstein, 1992). Suppose $v_{i}$ satisfies the singlecrossing property for each $i \in \mathcal{I}$. A mechanism $(q, t)$ is DIC if and only if for each $i \in \mathcal{I}$ and $\mathbf{x} \in X$ (i) $v_{i x}\left(q\left(s, \mathbf{x}_{-i}\right), x_{i}\right)$ is non-decreasing in $s$ and (ii) agent $i$ 's utility can be expressed as

$$
u_{i}\left(x_{i}, \mathbf{x}_{-i}\right)=u_{i}\left(\underline{x}_{i}, \mathbf{x}_{-i}\right)+\int_{\underline{x}_{i}}^{x_{i}} v_{i x}\left(q\left(s, \mathbf{x}_{-i}\right), s\right) d s .
$$

\footnotetext{
${ }^{2}$ Our main results (Theorem 1 and 2 can also be extended to discrete types similar to Gershkov et al. (2013).
} 
The result follows from Proposition 1, 2, and 3 of Mookherjee and Reichelstein (1992) and, hence, we omit its proof here.

Similar to dominant strategy incentive compatibility, we want to characterize Bayesian incentive compatibility. To accomplish this we need an appropriate extension of the single-crossing property to Bayesian settings. Note that from the perspective of each agent, who knows only the distribution of other agent types, the allocation rule induces a probability distribution over possible outcomes. This naturally leads to the following definition.

DEFINITION 3. Function $v_{i}$ satisfies the average single-crossing property when for any pair of probability distributions $G, F \in \Delta(A)$ if there exists type $y$ such that $\int v_{i x}(a, y) d G>\int v_{i x}(a, y) d F$, then for any type $x \in X_{i}, \int v_{i x}(a, x) d G>\int v_{i x}(a, x) d F$.

The following proposition shows that given the average single-crossing property BIC mechanisms can be indeed characterized by a monotone expected marginal condition and an envelope formula: ${ }^{3}$

Proposition 2. Suppose $v_{i}$ satisfies the average single-crossing property for each $i \in \mathcal{I}$. A mechanism $(q, t)$ is BIC if and only if for each $i \in \mathcal{I}$ and $x_{i} \in X_{i}(i) E_{\mathbf{x}_{-i}} v_{i x}\left(q\left(s, \mathbf{x}_{-i}\right), x_{i}\right)$ is non-decreasing in $s$ and (ii) agent $i$ 's interim expected utility can be expressed as

$$
U_{i}\left(x_{i}\right)=U_{i}\left(\underline{x}_{i}\right)+\int_{\underline{x}_{i}}^{x_{i}} E_{\mathbf{x}_{-i}} v_{i x}\left(q\left(s, \mathbf{x}_{-i}\right), s\right) d s
$$

The version of the single-crossing property due to Mookherjee and Reichelstein (1992) is arguably more restrictive than the standard strict (smooth) single-crossing differences condition, as in Milgrom (2004). We use the former property and its extension to Bayesian settings because the set of allocations $A$ and the set of distributions over allocations $\Delta(A)$ do not have an inherent order. Hence, the standard characterization of incentive compatibility, as in Milgrom (2004), does not apply to our settings.

The average single-crossing property gives us a readily workable characterization of Bayesian incentive compatibility. This property is, however, novel and we want to understand how it restricts agents' utilities before proceeding with further analysis. First of all, if the feasible set $A$ is the set of

\footnotetext{
${ }^{3}$ Note that the sufficiency part holds even without imposing the average single-crossing property.
} 
all possible lotteries over some set of alternatives, the average single-crossing and the single-crossing are equivalent because in this case any probability distribution over $A$ simply defines a compound lottery over the underlying set of alternatives. In general, however, the average single-crossing only implies the single-crossing. To see this, simply note that one can always consider a pair of deterministic distributions in the definition of the average single-crossing. Finally, we provide a full characterization of utilities that satisfy the average single-crossing property:

Proposition 3. A continuously differentiable function $v_{i}\left(a, x_{i}\right)$ with respect to agent's type satisfies the average single-crossing property if and only if

$$
v_{i}\left(a, x_{i}\right)=f_{i}(a) M_{i}\left(x_{i}\right)+m_{i}\left(x_{i}\right)+g_{i}(a),
$$

where $f_{i}$ and $g_{i}$ are continuous functions defined on the set of allocations $A ; M_{i}$ and $m_{i}$ are continuously differentiable functions with $M_{i}$ being either constant or increasing everywhere on $X_{i}$.

Given this result, we assume in the remainder of the paper that agent $i$ 's value function $v_{i}$ takes the form of (3) for each $i \in \mathcal{I}$. With this specification, the monotonicity conditions in the characterizations of DIC and BIC mechanisms are now equivalent to $f_{i}\left(q\left(s, \mathbf{x}_{-i}\right)\right)$ being non-decreasing in $s$ for each $i \in \mathcal{I}$ and $\mathbf{x}_{-i} \in X_{-i}$ and $E_{\mathbf{x}_{-i}} f_{i}\left(q\left(s, \mathbf{x}_{-i}\right)\right)$ being non-decreasing in $s$ for each $i \in \mathcal{I}$ respectively.

\section{The BIC-DIC Equivalence}

We use the following logic to prove the equivalence between Bayesian and dominant strategy implementation. For connected type spaces, the characterizations of DIC and BIC mechanisms (Propositions 1 and 2 imply that the interim expected utilities of agents are determined (up to a constant) by the allocation rule. Therefore, to match agents' interim expected utilities we basically need to match interim expected marginals $E_{\mathbf{x}_{-i}} f_{i}\left(q\left(x_{i}, \mathbf{x}_{-i}\right)\right)$ for each $i \in \mathcal{I}$ and $x_{i} \in X_{i}$. We also need to make sure that the equivalent mechanisms generate the same expected social surplus. In addition, to respect the incentive compatibility we need to satisfy the corresponding monotonicity conditions.

To state our main result, we introduce first the notion of convex-valued mappings. A mapping $\mathbf{f}: A \rightarrow \mathbb{R}^{I}$ with $\mathbf{f}(a)=\left(f_{1}(a), \ldots, f_{I}(a)\right)$ is convex-valued if its image is convex, i.e. for any $a, b \in A$ 
and $\alpha \in[0,1]$ there exists $c \in A$ such that $\mathbf{f}(c)=\alpha \mathbf{f}(a)+(1-\alpha) \mathbf{f}(b)$. We also note that for mappings $\mathbf{g}=\left(g_{1}, \ldots, g_{I}\right)$ and $\mathbf{f}=\left(f_{1}, \ldots, f_{I}\right)$ in $(3)$, if mapping $\mathbf{g}$ is a linear transformation of $\mathbf{f}$, then $\mathbf{f}$ is convex-valued if and only if the mapping of all agents utilities $\left(v_{1}\left(\cdot, x_{1}\right)+t_{1}, \ldots, v_{I}\left(\cdot, x_{I}\right)+t_{I}\right)$ is convex-valued for each $\left(x_{1}, \ldots, x_{I}\right) \in X: 4$

THEOREM 1. Assume mapping $\mathbf{f}$ is continuous and convex-valued, and $\mathbf{g}$ is a linear transformation of f. Then for any BIC mechanism $(\tilde{q}, \tilde{t})$ there exists an equivalent DIC mechanism $(q, t)$.

The proof of the theorem follows the main steps of the proof of Theorem 1 in Gershkov et al. (2013). The main part of the argument establishes that for a given BIC allocation rule $\tilde{q}$ there exists a feasible allocation $q$ that satisfies

$$
E_{\mathbf{x}_{-i}} f_{i}\left(q\left(x_{i}, \mathbf{x}_{-i}\right)\right)=E_{\mathbf{x}_{-i}} f_{i}\left(\tilde{q}\left(x_{i}, \mathbf{x}_{-i}\right)\right), \forall i \in \mathcal{I}, \forall x_{i} \in X_{i}
$$

and that has non-decreasing marginals $f_{i}\left(q\left(\cdot, \mathbf{x}_{-i}\right)\right)$ for all $i \in \mathcal{I}$ and $\mathbf{x}_{-i} \in X_{-i}$. We prove this statement for discrete and uniformly distributed types in Lemma 1 below. In particular, we develop an algorithm that finds a feasible allocation that satisfies (4) and that has non-decreasing marginals $5^{5}$ We then extend the proof to continuous types and arbitrary type distributions (see Lemmas A1 and A2). Finally, we construct transfers that lead to the same interim expected utilities and generate the same expected social surplus using the envelope formula (see Proposition 1).

Lemma 1. Suppose, for all $i \in \mathcal{I}, X_{i}$ is a discrete set and $\lambda_{i}$ is the uniform distribution on $X_{i}$. For any BIC allocation $\tilde{q}$ there exists a feasible allocation $q$ satisfying (4) and $f_{i}\left(q\left(\cdot, \mathbf{x}_{-i}\right)\right)$ being non-decreasing for all $i \in \mathcal{I}$ and $\mathbf{x}_{-i} \in X_{-i}$.

ProOF. Consider an arbitrary BIC allocation $\tilde{q}$, and let us assume $f_{j}\left(\tilde{q}\left(\cdot, \mathbf{x}_{-j}\right)\right)$ is not non-decreasing for some $j$ and $\mathbf{x}_{-j}$; otherwise the statement is trivial. Then, there exists some $x_{j}^{\prime}>x_{j}$ such that $f_{j}\left(\tilde{q}\left(x_{j}^{\prime}, \mathbf{x}_{-j}\right)\right)<f_{j}\left(\tilde{q}\left(x_{j}, \mathbf{x}_{-j}\right)\right)$. Since agent $j$ 's expected marginal $E_{\mathbf{x}_{-j}} f_{j}\left(\tilde{q}\left(\cdot, \mathbf{x}_{-j}\right)\right)$ is non-decreasing there also exists a set of other agents' types $X_{-j}^{\prime}$ such that $f_{j}\left(\tilde{q}\left(x_{j}^{\prime}, \mathbf{x}_{-j}^{\prime}\right)\right)>f_{j}\left(\tilde{q}\left(x_{j}, \mathbf{x}_{-j}^{\prime}\right)\right)$ for all

\footnotetext{
${ }^{4}$ The necessity part actually holds only under an additional mild condition. If we denote the matrix transforming f to $\mathbf{g}$ as $A$ and the diagonal matrix with elements $M_{i}\left(x_{i}\right)$ as $M(\mathbf{x})$ with $\mathbf{x}=\left(x_{1}, \ldots, x_{I}\right)$, the additional condition states: the sum of matrices $M(\mathbf{x})+A$ has a full rank.

${ }^{5}$ Gershkov et al. (2013) use a minimization problem to find a feasible allocation that satisfies (4) and that has non-decreasing marginals. Their minimization problem could also be adapted to our settings. We use an algorithmic proof because of its convenience in the proofs of our Theorem 2 and the applications presented in Section 5 .
} 
$\mathbf{x}_{-j}^{\prime} \in X_{-j}^{\prime}$. Now consider a new allocation $\hat{q} \neq \tilde{q}$ such that

$\mathbf{f}\left(\hat{q}\left(x_{j}, \mathbf{x}_{-j}\right)\right)=\frac{1}{2} \mathbf{f}\left(\tilde{q}\left(x_{j}, \mathbf{x}_{-j}\right)\right)+\frac{1}{2} \mathbf{f}\left(\tilde{q}\left(x_{j}^{\prime}, \mathbf{x}_{-j}\right)\right), \mathbf{f}\left(\hat{q}\left(x_{j}, \mathbf{x}_{-j}^{\prime}\right)\right)=(1-\delta) \mathbf{f}\left(\tilde{q}\left(x_{j}, \mathbf{x}_{-j}^{\prime}\right)\right)+\delta \mathbf{f}\left(\tilde{q}\left(x_{j}^{\prime}, \mathbf{x}_{-j}^{\prime}\right)\right)$,

$\mathbf{f}\left(\hat{q}\left(x_{j}^{\prime}, \mathbf{x}_{-j}\right)\right)=\frac{1}{2} \mathbf{f}\left(\tilde{q}\left(x_{j}^{\prime}, \mathbf{x}_{-j}\right)\right)+\frac{1}{2} \mathbf{f}\left(\tilde{q}\left(x_{j}, \mathbf{x}_{-j}\right)\right), \mathbf{f}\left(\hat{q}\left(x_{j}^{\prime}, \mathbf{x}_{-j}^{\prime}\right)\right)=(1-\delta) \mathbf{f}\left(\tilde{q}\left(x_{j}^{\prime}, \mathbf{x}_{-j}^{\prime}\right)\right)+\delta \mathbf{f}\left(\tilde{q}\left(x_{j}, \mathbf{x}_{-j}^{\prime}\right)\right)$,

for all $\mathbf{x}_{-j}^{\prime} \in X_{-j}^{\prime}$ and $\hat{q}(\mathbf{x})=\tilde{q}(\mathbf{x})$ for all other $\mathbf{x} \in \mathbf{X}$, where

$$
\delta=\frac{1}{2}\left(f_{j}\left(\tilde{q}\left(x_{j}, \mathbf{x}_{-j}\right)\right)-f_{j}\left(\tilde{q}\left(x_{j}^{\prime}, \mathbf{x}_{-j}\right)\right)\right) / \sum_{\mathbf{x}_{-j}^{\prime} \in X_{-j}^{\prime}}\left(f_{j}\left(\tilde{q}\left(x_{j}^{\prime}, \mathbf{x}_{-j}^{\prime}\right)\right)-f_{j}\left(\tilde{q}\left(x_{j}, \mathbf{x}_{-j}^{\prime}\right)\right)\right) .
$$

Since $E_{\mathbf{x}_{-j}} f_{j}\left(\tilde{q}\left(\cdot, \mathbf{x}_{-j}\right)\right)$ is non-decreasing we have $0 \leq \delta \leq \frac{1}{2}$. In addition, a feasible allocation $\hat{q}$ with $\hat{q}(\mathbf{x}) \in A$, for each $\mathbf{x} \in X$, is guaranteed to exist because mapping $\mathbf{f}$ is convex-valued. Equation (5) guarantees that the equal expected marginal condition (4) is satisfied for agent $j$ having types $x_{j}$ and $x_{j}^{\prime}$. For agent $j$ having other types, condition (4) follows trivially. For agent $i, i \neq j$, condition (4) follows from $\mathbf{f}\left(\hat{q}\left(x_{j}, \mathbf{x}_{-j}\right)\right)+\mathbf{f}\left(\hat{q}\left(x_{j}^{\prime}, \mathbf{x}_{-j}\right)\right)=\mathbf{f}\left(\tilde{q}\left(x_{j}, \mathbf{x}_{-j}\right)\right)+\mathbf{f}\left(\tilde{q}\left(x_{j}^{\prime}, \mathbf{x}_{-j}\right)\right)$ and $\mathbf{f}\left(\hat{q}\left(x_{j}, \mathbf{x}_{-j}^{\prime}\right)\right)+\mathbf{f}\left(\hat{q}\left(x_{j}^{\prime}, \mathbf{x}_{-j}^{\prime}\right)\right)=\mathbf{f}\left(\tilde{q}\left(x_{j}, \mathbf{x}_{-j}^{\prime}\right)\right)+\mathbf{f}\left(\tilde{q}\left(x_{j}^{\prime}, \mathbf{x}_{-j}^{\prime}\right)\right)$.

Let us now define $\hat{s}=E_{\mathbf{x}}\left(\| \mathbf{f}\left(\hat{q}(\mathbf{x}) \|^{2}\right)\right.$ and $\tilde{s}=E_{\mathbf{x}}\left(\| \mathbf{f}\left(\tilde{q}(\mathbf{x}) \|^{2}\right)\right.$, where $\|\cdot\|$ denotes the Euclidean norm $\|\mathbf{f}(q(\mathbf{x}))\|^{2}=\sum_{i \in \mathcal{I}} f_{i}(q(\mathbf{x}))^{2}$. Taking into account that $\lambda_{i}$ is uniformly distributed, we have

$\hat{s}-\tilde{s}=\left(-1 / 2|| \mathbf{f}\left(\tilde{q}\left(x_{j}, \mathbf{x}_{-j}\right)\right)-\mathbf{f}\left(\tilde{q}\left(x_{j}^{\prime}, \mathbf{x}_{-j}\right)\right)\left\|^{2}-2 \delta(1-\delta)\right\| \mathbf{f}\left(\tilde{q}\left(x_{j}^{\prime}, \mathbf{x}_{-j}^{\prime}\right)\right)-\mathbf{f}\left(\tilde{q}\left(x_{j}, \mathbf{x}_{-j}^{\prime}\right)\right) \|^{2}\right) /|X|<0$

If $f_{j}\left(\hat{q}\left(\cdot, \mathbf{x}_{-j}\right)\right)$ is not non-decreasing for some $j$ and $\mathbf{x}_{-j}$, we repeat the above procedure. Iterating the procedure we finally obtain a sequence of allocations $q^{n} \in A$ and a sequence of values $s^{n} \geq 0$ for $n=1,2, \ldots$. If for some $n$ we find that $f_{j}\left(q^{n}\left(\cdot, \mathbf{x}_{-j}\right)\right)$ is non-decreasing for all $j$ and $\mathbf{x}_{-j}$ we set $q^{n+1} \equiv q^{n}$ and $s^{n+1} \equiv s^{n}$. By construction, $s^{n}$ is a weakly decreasing sequence that is bounded below by 0 . Hence, $s^{n}$ has a limit that we denote as $s$. Since set $A$ is compact there exists also a convergent subsequence of $q^{n}$ with a limit $q$ such that $q(\mathbf{x}) \in A$ for all $\mathbf{x} \in X$. By construction, $s=E_{\mathbf{x}}\left(\| \mathbf{f}\left(q(\mathbf{x}) \|^{2}\right)\right.$ and $f_{j}\left(q\left(\cdot, \mathbf{x}_{-j}\right)\right)$ are non-decreasing for all $j$ and $\mathbf{x}_{-j}$.

Proof of Theorem 1. Lemmas A1 and A2 in the Appendix extend Lemma 1 to show that, given any set $X_{i} \subset \mathbb{R}$ and any distribution $\lambda_{i}$, for any BIC allocation $\tilde{q}$ there exists a feasible allocation $q$ satisfying (4) with non-decreasing marginals $f_{i}\left(q\left(\cdot, \mathbf{x}_{-i}\right)\right)$ for all $i \in \mathcal{I}$ and $\mathbf{x}_{-i} \in X_{-i}$. Since these 
lemmas are rather technical we postpone them to the Appendix. To finish the construction of an equivalent DIC mechanism we now consider transfers $t$ defined by

$$
t_{i}\left(x_{i}, \mathbf{x}_{-i}\right)=t_{i}\left(\underline{x}_{i}, \mathbf{x}_{-i}\right)+v_{i}\left(q\left(\underline{x}_{i}, \mathbf{x}_{-i}\right), \underline{x}_{i}\right)-v_{i}\left(q\left(x_{i}, \mathbf{x}_{-i}\right), x_{i}\right)+\int_{\underline{x}_{i}}^{x_{i}} v_{i x}\left(q\left(s, \mathbf{x}_{-i}\right), s\right) d s
$$

for all $\mathbf{x} \in X, i \in \mathcal{I}$, where $t_{i}\left(\underline{x}_{i}, \mathbf{x}_{-i}\right)=E_{\mathbf{x}_{-i}}\left(v_{i}\left(\tilde{q}\left(\underline{x}_{i}, \mathbf{x}_{-i}\right), \underline{x}_{i}\right)+\tilde{t}_{i}\left(\underline{x}_{i}, \mathbf{x}_{-i}\right)\right)-v_{i}\left(q\left(\underline{x}_{i}, \mathbf{x}_{-i}\right), \underline{x}_{i}\right)$. Proposition 1 guarantees that mechanism $(q, t)$ is DIC. In addition, mechanism $(q, t)$ leads to the same interim expected utilities as in BIC mechanism $(\tilde{q}, \tilde{t})$. In particular,

$$
\begin{aligned}
U_{i}\left(x_{i}\right) & =E_{\mathbf{x}_{-i}}\left(\tilde{t}_{i}\left(\underline{x}_{i}, \mathbf{x}_{-i}\right)+v_{i}\left(\tilde{q}\left(\underline{x}_{i}, \mathbf{x}_{-i}\right), \underline{x}_{i}\right)\right)+\int_{\underline{x}_{i}}^{x_{i}} E_{\mathbf{x}_{-i}} v_{i x}\left(q\left(s, x_{-i}\right), s\right) d s \\
& =E_{\mathbf{x}_{-i}}\left(\tilde{t}_{i}\left(\underline{x}_{i}, \mathbf{x}_{-i}\right)+v_{i}\left(\tilde{q}\left(\underline{x}_{i}, \mathbf{x}_{-i}\right), \underline{x}_{i}\right)\right)+\int_{\underline{x}_{i}}^{x_{i}} E_{\mathbf{x}_{-i}} v_{i x}\left(\tilde{q}\left(s, x_{-i}\right), s\right) d s=\tilde{U}_{i}\left(x_{i}\right),
\end{aligned}
$$

where the first equality follows from (6), the second one from (4), and the third one from the characterization of BIC mechanisms (Proposition 2). When mapping $\mathbf{g}$ is a linear transformation of $\mathbf{f}$, the equal expected marginal conditions in (4) also imply $E_{\mathbf{x}}\left[\sum_{i \in \mathcal{I}} g_{i}(q(\mathbf{x}))\right]=E_{\mathbf{x}}\left[\sum_{i \in \mathcal{I}} g_{i}(\tilde{q}(\mathbf{x}))\right]$. Hence, both mechanisms also generate the same expected social surplus.

Theorem 1 extends the BIC-DIC equivalence result to non-linear environments when each agent's utility satisfies the average single-crossing property and the mapping of all agents' utilities is convexvalued. In addition, the new proof only requires the set of feasible allocations $A$ being compact instead of being a simplex as in Gershkov et al. (2013).

The requirement that $\mathbf{g}$ is a linear transformation of $\mathbf{f}$ is satisfied, for example, if all terms $g_{i}$ are zero as in some applications of Section 5. For general $\mathrm{g}$ the constructed DIC mechanism, however, does not necessarily match the expected social surplus of the BIC mechanism 6 We now provide sufficient conditions when for a given BIC mechanism one could find a DIC mechanism that produces the same interim expected utilities and generates at least as large expected social surplus.

For this purpose, we consider environments where the set of feasible allocations $A$ is a convex and compact subset of $\mathbb{R}^{I}$ with $q=\left(q_{1}, \ldots, q_{I}\right)$, where $q_{i} \in \mathbb{R}$ for each $i \in \mathcal{I}$. We also assume that functions $f_{i}$ depend on different components of allocations, i.e. $f_{i}(q)=\check{f}_{i}\left(q_{i}\right)$, for all $i \in \mathcal{I}, q \in A$.

\footnotetext{
${ }^{6}$ For general $\mathbf{g}$ the constructed DIC mechanism still delivers the same interim expected utilities for all agents.
} 
TheOREM 2. Assume mapping $\mathbf{f}$ is continuous and convex-valued. For any BIC mechanism there exists a DIC mechanism that delivers the same interim expected utilities for all agents. In addition, the DIC mechanism generates at least as large expected social surplus, if for each $i \in \mathcal{I}$

(i) $\check{f}_{i}\left(q_{i}\right)$ is non-decreasing and concave (or non-increasing and convex) and $g_{i}(q)$ is continuous, non-increasing, and concave in each component, or

(ii) $\check{f}_{i}\left(q_{i}\right)$ is non-increasing and concave (or non-decreasing and convex) and $g_{i}(q)$ is continuous, non-decreasing, and concave in each component.

Remark. The theorem also extends to settings where the set of feasible allocations $A$ is compact, mapping $\mathbf{f}$ is continuous and convex-valued, and the utility of each agent satisfies the following condensation property. Functions $f_{i}$ and $g_{i}$ can be written as $f_{i}(q)=\check{f}_{i}\left(h_{i}(q)\right)$ and $\sum_{i} g_{i}(q)=$ $G\left(h_{1}(q), \ldots, h_{I}(q)\right)$ for all $q \in A$, where $h_{i}: A \rightarrow \mathbb{R}, \check{f}_{i}$ is non-decreasing and concave (or nonincreasing and convex), and the aggregate function $G: \mathbb{R}^{I} \rightarrow \mathbb{R}$ is continuous, non-increasing, and concave in each component.7 The proof of this extension repeats the steps of the proof of Theorem 2 presented in the Appendix, and we omit it to avoid repetition. We exploit this observation when we consider the environmental mechanism design applications in Section 5.

We want to note that Theorem 2 demands a DIC mechanism to produce only at least as large expected social surplus as in the original BIC mechanism. This requirement is more flexible than the one in the definition of equivalent mechanisms (see Definition 1). In addition, it better captures the economic intuition that one does not need to insert additional money to achieve a more robust solution concept. In particular, a mechanism designer concerned with revenue-maximization extracts a higher level of revenue with the constructed DIC mechanism. Hence, this requirement should have a broader range of meaningful economic applications, which we also illustrate in Section 5 .

\section{Applications}

In this section, we demonstrate that Theorem 1 and 2 apply to many important environments where previous works have little bite (e.g. Manelli and Vincent, 2010; Gershkov et al., 2013). In addition, they produce several novel implications that are of independent interest.

\footnotetext{
${ }^{7}$ Similar to condition (ii) in the theorem the result also extends to settings when $\check{f}_{i}$ is non-increasing and concave (or non-decreasing and convex) and $G$ is continuous, non-decreasing, and concave in each component.
} 


\subsection{Principal-Agent Problem and Allocative Externalities}

Consider a standard contracting setting where a principal needs to procure $I$ goods from $I$ agents. Assume the principal chooses a production plan $q=\left(q_{1}, \ldots, q_{I}\right) \in A \equiv \prod_{i=1}^{I}\left[\underline{q}_{i}, \bar{q}_{i}\right] \subseteq \mathbb{R}^{I}$ and a transfer scheme $\left(t_{1}, \ldots, t_{I}\right) \in \mathbb{R}^{I}$, the payoff of agent $i$ is then given by $-c_{i}\left(q_{i}\right) x_{i}+t_{i}$, where $c_{i}:\left[\underline{q}_{i}, \bar{q}_{i}\right] \rightarrow \mathbb{R}$ is some continuous non-decreasing function with an interpretation of $c_{i}\left(q_{i}\right) x_{i}$ being agent $i$ 's cost of supplying $q_{i}$ units of good $i$. Many influential papers analyzing the optimal procurement contracts fall into this setting (e.g., Laffont and Martimort, 1997; Mookherjee and Tsumagari, 2004; Severinov, 2008; Duenyas, et al., 2013). In this setting, we have $f_{i}(q)=-c_{i}\left(q_{i}\right)$ and $g_{i}(q)=0$ for each $i \in \mathcal{I}$. Since functions $c_{i}$ are continuous the Intermediate Value Theorem 8 implies that mapping $\mathbf{f}(\cdot)=\left(-c_{1}(\cdot), \ldots,-c_{I}(\cdot)\right)$ is convex-valued. Then, Theorem 1 leads to the following corollary.

Corollary 1. Consider the standard procurement setting. If $c_{i}$ is continuous for $i \in \mathcal{I}$, for any $B I C$ mechanism there exists an equivalent DIC mechanism.

In many contracting situations, agents may care not only about their own contracts with the principal, but also have preferences over the contracts received by other agents. For instance, a country may prefer its ally rather than its enemy to get a weapon contract (see Jehiel et al., 1996). Similar concerns arise in the presence of downstream competition among the firms after the allocation of contracts (Segal, 1999) 9 W Within the current framework, type-independent allocative externalities can be captured by incorporating an additional term into agent's utility function, i.e. $-c_{i}\left(q_{i}\right) x_{i}+g_{i}(q)+t_{i}$. Assuming that the cost and externality functions satisfy the conditions of Theorem 2, we establish the following result.

Corollary 2. Consider a procurement setting with allocation externalities. If $c_{i}$ is continuous for each $i \in \mathcal{I}$, then for any BIC mechanism there exists a DIC mechanism that delivers the same interim expected utilities for all agents. If $c_{i}$ is also non-decreasing and convex (concave) and $g_{i}$ is continuous, non-decreasing (non-increasing), and concave in each component for each $i \in \mathcal{I}$, then the DIC mechanism generates at least as large expected social surplus as the BIC mechanism.

Corollary 2 identifies environments with allocative externalities, where a mechanism designer can

\footnotetext{
${ }^{8}$ See, for example, Chapter 2 of Royden (1988).

${ }^{9}$ See Jehiel and Moldovanu (2006) for an excellent overview of papers analyzing allocative externalilities.
} 
rely on dominant strategy implementation and gains nothing from designing more complex BIC mechanisms. This is in sharp contrast to results pertaining to environments with both allocative and information externalities, where more robust solution concepts appear to be much more restrictive (see Jehiel and Moldovanu, 2006).

\subsection{Mechanism Design for the Environment}

Let us first consider a model of Martimort and Sand-Zantman (2013, 2015) who analyze feasible agreements in reducing the aggregate pollution of $I$ countries. Each country $i$ can exert effort $q_{i} \in[\underline{q}, \bar{q}] \subseteq \mathbb{R}_{+}$that have both local benefits of size $\alpha q_{i}$ (with $\alpha \in[0,1)$ ) and global benefits of size $(1-\alpha) q_{i}$, which accrue worldwide. The countries differ in their costs of effort $q_{i}^{2} x_{i} / 2$ with $x_{i}$ being country $i$ 's efficiency parameter. Efficiency parameters are drawn independently from the same cumulative distribution $\lambda$ with support $[\underline{x}, \bar{x}] \subseteq \mathbb{R}$. Overall, country $i$ 's payoff is given by $-q_{i}^{2} x_{i} / 2+\alpha q_{i}+(1-\alpha) Q+t_{i}$, where $Q=\sum_{i=1}^{I} q_{i}$ is aggregate global benefits and $t_{i}$ is a monetary transfer to country $i$. Taking into account that function $-q_{i}^{2} / 2$ is non-increasing and concave and the externality function $\alpha q_{i}+(1-\alpha) Q$ is non-decreasing and linear (and, hence, concave), the following result directly follows from Theorem 2 .

Corollary 3. Consider the environmental mechanism design setting of Martimort and SandZantman (2013, 2015). Then, for any BIC mechanism there exists a DIC mechanism producing the same interim expected utilities to all agents and generating at least as large expected social surplus.

Baliga and Maskin (2003) also study feasible agreements to efficiently reduce the aggregate pollution level, but consider a slightly different model. While they assume that agents' costs are type-independent, agents have private information about their value of the pollution reduction. More specifically, agent $i$ 's utility is given by $x_{i} Q^{1 / 2}-q_{i}+t_{i}$, where $x_{i} Q^{1 / 2}$ is the gross benefits to agent $i$ from aggregate reduction $Q$. Though Theorem 2 does not formally apply to this environment, each agent $i$ 's benefits and costs from pollution reduction satisfy the condensation property defined in the remark after Theorem 2. In particular, agent $i$ 's benefits equal $\check{f}_{i}\left(h_{i}(q)\right)=h_{i}(q)^{\frac{1}{2}}$ and the aggregate costs equal $\sum_{i \in \mathcal{I}} q_{i}=\sum_{i \in \mathcal{I}} q_{i}=-G\left(h_{1}(q), \ldots, h_{I}(q)\right)$, where the condensation function $h_{i}(q)=\sum_{i \in \mathcal{I}} q_{i}, i \in \mathcal{I}$, is the same for all agents. Note that mapping $\mathbf{f}=\left(f_{1}(\cdot), \ldots, f_{I}(\cdot)\right)$ is 
symmetric and, hence, the Intermediate Value Theorem implies that it is convex-valued. In addition, $\check{f}_{i}$ is non-decreasing and concave, and function $G$ is non-increasing and linear (and, hence, concave). Hence, the following result is implied by the extension discussed in the remark after Theorem 2 .

COROllary 4. Consider the environmental mechanism design setting of Baliga and Maskin (2003). Then, for any BIC mechanism there exists a DIC mechanism producing the same interim expected utilities to all agents and generating at least as large expected social surplus.

Corollaries 3 and 4 imply that the mechanism designer would lose nothing by restricting himself to DIC mechanisms for environmental design problems, if he/she wants to maintain the same level of agents' interim expected utility without the influx of additional money into the system. This result, however, holds without imposing additional constraints - such as ex post budget balance, as thoroughly discussed in Baliga and Maskin (2003). Though Bayesian implementation is more permissive when ex post budget balance is imposed, the mechanism designer can still rely only on DIC mechanisms if the budget balance constraint needs to be satisfied in expectations. We show this result in the next application.

\subsection{Public Good Provision}

Consider a standard setting of public good provision with $I \geq 2$ agents. If $q \in A=[\underline{q}, \bar{q}]$ units of public good is provided, agent $i$ 's utility is given by $f(q) x_{i}+t_{i}$, where $f(q) x_{i}$ is agent $i$ 's valuation of the public good and $t_{i} \in \mathbb{R}$ is the units of private good that he receives. Many influential papers on public good provision fall into this setting (e.g., Mailath and Postlewaite (1990), Ledyard and Palfrey (1999), Hellwig (2003), Norman (2004)). If $f: A \rightarrow \mathbb{R}$ is continuous in $q$, it again follows from the Intermediate Value Theorem that the mapping $\mathbf{f}(\cdot)=(f(\cdot), \ldots, f(\cdot))$ is convex-valued and, hence, Theorem 1 can be applied here.

Corollary 5. Consider the public good provision setting. If $f$ is continuous, then for any BIC mechanism there exists an equivalent DIC mechanism.

While the equivalent DIC mechanism, constructed in Theorem 1, inherits interim individual rationality from the BIC mechanism ${ }^{10}$ there is no guarantee that other constraints imposed on the

\footnotetext{
${ }^{10}$ The constructed DIC mechanism satisfies even a stronger notion of ex post individual rationality.
} 
BIC mechanism will remain satisfied as well. For example, when designing a mechanism for public good provision it is typical to require that the private goods raised from the agents are enough to cover the cost of the public good. Formally, a direct mechanism $(q, t)$ is ex ante budget balanced if

$$
\int_{\mathbf{x} \in X}\left[K(q(\mathbf{x}))+\sum_{i=1}^{I} t_{i}(\mathbf{x})\right] d \lambda(\mathbf{x}) \leq 0
$$

where $K: A \rightarrow \mathbb{R}$ is the cost function of producing the public good. The following corollary of Theorem 2 provides a sufficient condition under which the equivalent DIC mechanism constructed in Theorem 1 also inherits ex ante budget balance from the original BIC mechanism. ${ }^{11}$

Corollary 6. Suppose $f$ is continuous, non-decreasing, and concave and $K$ is continuous, nondecreasing, and convex. For any BIC mechanism that is ex ante budget balanced the equivalent DIC mechanism, constructed in Theorems 1, is also ex ante budget balanced.

Intuitively, the monotonicity and concavity of utility functions imply that the provision of public good is more balanced across states in the equivalent DIC mechanism than that in the BIC mechanism. Consequently, the expected cost of providing the public good is lower. Since the expected transfers remain unchanged in the equivalent DIC mechanism constructed in Theorem 1, the property of ex ante budget balance is preserved.

Our result thus suggests that for a quite general class of public good provision problems it is without loss of generality to insist on dominant-strategy incentive compatibility even when the additional ex ante budget balance constraint is imposed ${ }^{12}$ For example, the second-best allocation rule in Hellwig (2003) can be equivalently implemented in dominant strategies without violating the ex ante budget balance condition if functions $f$ and $K$ are concave and convex respectively.

\footnotetext{
${ }^{11}$ The result of Corollary 6 extends without any change to non-symmetric settings with mapping $\mathbf{f}=\left(f_{1}(\cdot), \ldots, f_{I}(\cdot)\right)$ being convex-valued and functions $f_{i}, i \in \mathcal{I}$, being continuous, non-decreasing, and concave.

${ }^{12}$ For some applications, it is natural to require mechanisms to be ex post budget balanced, i.e. inequality (8) holds for each $\mathbf{x} \in X$. Borgers and Norman (2009) show that for every ex ante budget balanced DIC mechanism $(q, t)$ there exist transfers $t^{\prime}$ such that $\left(q, t^{\prime}\right)$ is (i) BIC for all agents and DIC for all but one agent and (ii) ex post budget balanced. Agents also have the same interim expected payments in both mechanisms (see also Borgers, 2015, Ch. 3).
} 


\section{Conclusion}

This paper extends the equivalence between Bayesian and dominant strategy implementation to non-linear environments where each agent's utility satisfies the average single-crossing property and the mapping of all agents' utilities is convex-valued. These assumptions are satisfied by many important environments in the literature on principal-agent problems with allocative externalities, environmental mechanism design, and public good provision. Since the results of the previous papers (Manelli and Vincent, 2010; Gershkov et al., 2013) do not apply to these environments the current paper significantly enlarges the set of settings where the mechanism designer can rely on more robust solution concept of dominant strategy implementation.

In this paper, we also provide sufficient conditions when for a given BIC mechanism there exists a DIC mechanism that yields the same interim expected utilities to all agents and generates at least as large social surplus (see also Kushnir, 2015). Using this result we provide several novel implications for the above-mentioned environments. In addition, being more flexible than the notion of equivalence due to Gershkov et al. (2013), this way of comparing two implementation concepts broadens the set of environments when the mechanism designer could stick to a more robust notion of implementation without sacrificing his/her objectives. Hence, we believe this notion will be useful for future studies.

Finally, we want to outline a possible research agenda. In their original paper, Gershkov et al. (2013) provide examples showing that the BIC-DIC equivalence generally fails in environments with non-linear utilities, correlated or multi-dimensional types, and interdependent values. This paper, however, shows that the equivalence can be extended to important non-linear settings. Similarly,

Kushnir (2015) shows that the equivalence extends to interesting environments with correlated types. We believe that one should be able to identify the conditions when the BIC-DIC equivalence holds for the environments with multi-dimensional types and interdependent values. We leave this exciting prospect for future research. 


\section{Appendix}

\section{Proof of Proposition 2}

For the sufficiency part, note that agent $i$ does not deviate from the truth-telling Bayes-Nash equilibrium if and only if

$$
U_{i}\left(x_{i}\right) \geq E_{\mathbf{x}_{-i}}\left(v_{i}\left(q\left(x_{i}^{\prime}, \mathbf{x}_{-i}\right), x_{i}\right)+t_{i}\left(x_{i}^{\prime}, \mathbf{x}_{-i}\right)\right)=U_{i}\left(x_{i}^{\prime}\right)+E_{\mathbf{x}_{-i}}\left(v_{i}\left(q\left(x_{i}^{\prime}, \mathbf{x}_{-i}\right), x_{i}\right)-v_{i}\left(q\left(x_{i}^{\prime}, \mathbf{x}_{-i}\right), x_{i}^{\prime}\right)\right)
$$

for all $x_{i}, x_{i}^{\prime} \in X_{i}$. Using (2), this is equivalent to require that for all $x_{i}, x_{i}^{\prime} \in X_{i}$,

$$
\int_{x_{i}^{\prime}}^{x_{i}} E_{\mathbf{x}_{-i}}\left(v_{i x}\left(q\left(s, \mathbf{x}_{-i}\right), s\right)\right) d s \geq E_{\mathbf{x}_{-i}}\left(v_{i}\left(q\left(x_{i}^{\prime}, \mathbf{x}_{-i}\right), x_{i}\right)\right)-E_{\mathbf{x}_{-i}}\left(v_{i}\left(q\left(x_{i}^{\prime}, \mathbf{x}_{-i}\right), x_{i}^{\prime}\right)\right),
$$

which is true under the condition that $E_{\mathbf{x}_{-i}}\left(v_{i x}\left(q\left(s, \mathbf{x}_{-i}\right), x_{i}\right)\right)$ is non-decreasing in $s$ for all $x_{i} \in X_{i}$.

For the necessity part, suppose that mechanism $(q, t)$ is BIC. We then have

$$
U_{i}\left(x_{i}\right)=\max _{x_{i}^{\prime} \in X_{i}}\left(E_{\mathbf{x}_{-i}}\left(v_{i}\left(q\left(x_{i}^{\prime}, \mathbf{x}_{-i}\right), x_{i}\right)+t_{i}\left(x_{i}^{\prime}, \mathbf{x}_{-i}\right)\right)\right)
$$

Since $v_{i}$ is continuously differentiable on $\left[\underline{x}_{i}, \bar{x}_{i}\right]$ it is also absolutely continuous and has a bounded derivative $v_{i x}$. Hence, equation (2) follows from the envelope theorem (Milgrom and Segal, 2002). It remains to show that BIC also implies the monotone-expected-marginal condition. Suppose, in contradiction, $E_{\mathbf{x}_{-i}} v_{i x}\left(q\left(y, \mathbf{x}_{-i}\right), z\right)>E_{\mathbf{x}_{-i}} v_{i x}\left(q\left(x, \mathbf{x}_{-i}\right), z\right)$ for some agent $i$ and $x, y, z \in X_{i}$, with $y<x$. The average single-crossing property implies that

$$
\int_{y}^{x} E_{\mathbf{x}_{-i}} v_{i x}\left(q\left(y, \mathbf{x}_{-i}\right), s\right) d s>\int_{y}^{x} E_{\mathbf{x}_{-i}} v_{i x}\left(q\left(x, \mathbf{x}_{-i}\right), s\right) d s
$$

At the same time, the incentive compatibility implies

$$
E_{\mathbf{x}_{-i}}\left(v_{i}\left(q\left(y, \mathbf{x}_{-i}\right), x\right)-v_{i}\left(q\left(y, \mathbf{x}_{-i}\right), y\right)\right) \leq U_{i}(x)-U_{i}(y) \leq E_{\mathbf{x}_{-i}}\left(v_{i}\left(q\left(x, \mathbf{x}_{-i}\right), x\right)-v_{i}\left(q\left(x, \mathbf{x}_{-i}\right), y\right)\right) .
$$


Using equation (2) we then obtain

$$
\int_{y}^{x} E_{\mathbf{x}_{-i}}\left(v_{i x}\left(q\left(y, \mathbf{x}_{-i}\right), s\right)\right) d s \leq \int_{y}^{x} E_{\mathbf{x}_{-i}}\left(v_{i x}\left(q\left(s, \mathbf{x}_{-i}\right), s\right)\right) d s \leq \int_{y}^{x} E_{\mathbf{x}_{-i}}\left(v_{i x}\left(q\left(x, \mathbf{x}_{-i}\right), s\right)\right) d s .
$$

Hence, we reach a contradiction.

\section{Proof of Proposition 3}

The sufficiency part is straightforward. Let us prove the necessity part. Consider some $x_{i} \in X_{i}$ and let $\bar{m}_{i x}\left(x_{i}\right)=\max _{a \in A} v_{i x}\left(a, x_{i}\right)$ and $\underline{m}_{i x}\left(x_{i}\right)=\min _{a \in A} v_{i x}\left(a, x_{i}\right)$. Note that both $\bar{m}_{i x}$ and $\underline{m}_{i x}$ are well-defined since $v_{i}(a, x)$ is continuously differentiable. The average single-crossing property ensures that the maximum and the minimum are achieved for the same alternatives across types $x_{i}$. We denote them as $a_{\text {max }}$ and $a_{\text {min }}$ respectively. If $\bar{m}_{i x}\left(x_{i}\right)=\underline{m}_{i x}\left(x_{i}\right)$, the average single-crossing implies that for every $x_{i} \in X_{i}$ function $v_{i x}\left(\cdot, x_{i}\right)$ is constant and, hence, satisfies equation (3). Otherwise, for each $a^{\prime} \in A$, there exists a unique number $f_{i}\left(a^{\prime}\right) \in[0,1]$ such that $v_{i x}\left(a^{\prime}, x_{i}\right)=$ $f_{i}\left(a^{\prime}\right) \bar{m}_{i x}\left(x_{i}\right)+\left(1-f_{i}\left(a^{\prime}\right)\right) \underline{m}_{i x}\left(x_{i}\right)$. Consider a distribution $F \in \Delta(A)$ such that $a_{\max }$ is chosen with probability $f_{i}\left(a^{\prime}\right)$ and $a_{m i n}$ is chosen with probability $1-f_{i}\left(a^{\prime}\right)$. By construction, we have

$$
\int v_{i x}\left(a, x_{i}\right) d F=v_{i x}\left(a^{\prime}, x_{i}\right)=f_{i}\left(a^{\prime}\right) \bar{m}_{i x}\left(x_{i}\right)+\left(1-f_{i}\left(a^{\prime}\right)\right) \underline{m}_{i x}\left(x_{i}\right) .
$$

The average single-crossing property then implies that for any $y \in X_{i}$

$$
\int v_{i x}(a, y) d F=v_{i x}\left(a^{\prime}, y\right)=f_{i}\left(a^{\prime}\right) \bar{m}_{i x}(y)+\left(1-f_{i}\left(a^{\prime}\right)\right) \underline{m}_{i x}(y)
$$

Hence, for all $a \in A, x_{i} \in X_{i}$

$$
v_{i x}\left(a, x_{i}\right)=f_{i}(a) M_{i x}\left(x_{i}\right)+m_{i x}\left(x_{i}\right)
$$

where $m_{i x}\left(x_{i}\right)=\underline{m}_{i x}\left(x_{i}\right)$ and $M_{i x}\left(x_{i}\right)=\bar{m}_{i x}\left(x_{i}\right)-\underline{m}_{i x}\left(x_{i}\right)$. Integrating (A.1), we obtain (3). 


\section{Proof of Theorem 1}

Lemma A1. Suppose, for all $i \in \mathcal{I}, X_{i}=[0,1]$ and $\lambda_{i}$ is the uniform distribution on $X_{i}$. Then, for any BIC allocation $\tilde{q}$ there exists a feasible allocation $q$ satisfying (4) with $f_{i}\left(q\left(\cdot, \mathbf{x}_{-i}\right)\right)$ being non-decreasing for all $i \in \mathcal{I}$ and $\mathbf{x}_{-i} \in X_{-i}$.

ProOf. The proof essentially repeats the proof of Lemma 2 in Gershkov et al. (2013), and we only sketch it here. We consider a partition $[0,1]^{I}$ to $2^{n I}$ cubes of equal size. For each cube $S$ in this partition, we approximate $\mathbf{f}(\tilde{q}(\mathbf{x})), \mathbf{x} \in S$, by its average defined by

$$
\mathbf{f}(\tilde{q}(S))=2^{n I} \int_{S} \mathbf{f}(\tilde{q}(\mathbf{x})) d \mathbf{x}
$$

Note allocation $\tilde{q}(S) \in A$ is well-defined, because mapping $\mathbf{f}$ is convex-valued. In addition, discrete allocation $\tilde{q}(S)$ inherits non-decreasing expected marginals from $\tilde{q}$. Lemma 1 then ensures that there exists an allocation $q(S)$ with non-decreasing marginals that can also be extended to piecewise constant functions over $[0,1]^{I}$. Taking the limit with respect to the size of partition, we obtain the result of the lemma. For the details of the construction, we refer to Gershkov et al. (2013).

Lemma A2. Suppose, for all $i \in \mathcal{I}, X_{i} \subseteq \mathbb{R}$ and $\lambda_{i}$ is some distribution on $X_{i}$. Then, for any BIC allocation $\tilde{q}$ there exists a feasible allocation $q$ satisfying (4) with $f_{i}\left(q\left(\cdot, \mathbf{x}_{-i}\right)\right)$ being non-decreasing for all $i \in \mathcal{I}$ and $\mathbf{x}_{-i} \in X_{-i}$.

Proof. The proof repeats the proof of Lemma 3 in Gershkov et al. (2013). Its main idea is to relate the uniform distribution covered by Lemma A1 to the case of a general distribution. In particular, if random variable $Z_{i}$ is uniformly distributed then $\lambda_{i}^{-1}\left(Z_{i}\right)$ is distributed according to $\lambda_{i} \cdot{ }^{13}$ Hence, for a given $\mathrm{BIC}$ allocation $\tilde{q}$ we use transformation $\lambda_{i}^{-1}$ to construct an allocation $\tilde{q}^{\prime}$ defined on uniformly distributed types that has also a non-decreasing expected marginals. For allocation $\tilde{q}^{\prime}$, we then apply the results of Lemmas 1 and A1 to obtain an allocation $q^{\prime}$ with non-decreasing marginals defined on uniformly distributed types. We then use transformation $\lambda_{i}$ to recover back an allocation $q$ with non-decreasing marginals defined on types distributed according to $\lambda_{i}$. For the details of the construction, we refer to Gershkov et al. (2013).

\footnotetext{
${ }^{13}$ Where $\lambda_{i}^{-1}\left(z_{i}\right)=\inf \left\{x_{i} \in X_{i} \mid \lambda_{i}\left(x_{i}\right) \geq z_{i}\right\}$.
} 


\section{Proof of Theorem 2}

Consider an arbitrary BIC mechanism $(\tilde{q}, \tilde{t})$ and the corresponding DIC mechanism $(q, t)$ constructed in Theorem 1. Since equation (7) holds for any $\mathbf{g}$, the first part of Theorem 2 immediately follows. The idea behind the proof of the second part of theorem is to show that if functions $\check{f}_{i}$ and $g_{i}$ satisfy conditions (i) or (ii), the DIC mechanism constructed in Theorem 1 also satisfies

$$
E_{\mathbf{x}}\left(\sum_{i} g_{i}(q(\mathbf{x}))\right) \geq E_{\mathbf{x}}\left(\sum_{i} g_{i}(\tilde{q}(\mathbf{x}))\right)
$$

Suppose condition (i) is satisfied. Let us first consider the case where types are discrete and uniformly distributed (as in Lemma 1). If the marginals of allocation $\tilde{q}$ are not non-decreasing, then $\check{f}_{j}\left(\tilde{q}_{j}\left(x_{j}^{\prime}, \mathbf{x}_{j}\right)\right)<\check{f}_{j}\left(\tilde{q}_{j}\left(x_{j}, \mathbf{x}_{-j}\right)\right)$ for some $j, x_{j}^{\prime}>x_{j}$, and $\mathbf{x}_{-j}$. Using the construction of the algorithm in Lemma 1 we then obtain an allocation $\hat{q} \in A$ satisfying the equal-marginal conditions in (4) and delivering strictly smaller value to objective $E_{\mathbf{x}}\|\mathbf{f}(\cdot)\|^{2}$. Since function $\check{f}_{j}$ is non-decreasing and concave (or non-increasing and convex), we also have

$$
\begin{aligned}
\hat{q}_{j}\left(x_{j}, \mathbf{x}_{-j}\right)= & \hat{q}_{j}\left(x_{j}^{\prime}, \mathbf{x}_{-j}\right) \leq \frac{1}{2} \tilde{q}_{j}\left(x_{j}, \mathbf{x}_{-j}\right)+\frac{1}{2} \tilde{q}_{j}\left(x_{j}^{\prime}, \mathbf{x}_{-j}\right), \\
& \hat{q}_{j}\left(x_{j}, \mathbf{x}_{-j}^{\prime}\right) \leq(1-\delta) \tilde{q}_{j}\left(x_{j}, \mathbf{x}_{-j}^{\prime}\right)+\delta \tilde{q}_{j}\left(x_{j}^{\prime}, \mathbf{x}_{-j}^{\prime}\right), \\
& \hat{q}_{j}\left(x_{j}^{\prime}, \hat{\mathbf{x}}_{-j}\right) \leq(1-\delta) \tilde{q}_{j}\left(x_{j}^{\prime}, \mathbf{x}_{-j}^{\prime}\right)+\delta \tilde{q}_{j}\left(x_{j}, \mathbf{x}_{-j}^{\prime}\right) .
\end{aligned}
$$

Since function $g_{i}$ is non-increasing and concave in each component, this further implies

$$
\begin{aligned}
& g_{i}\left(\hat{q}\left(x_{j}, \mathbf{x}_{-j}\right)\right)+g_{i}\left(\hat{q}\left(x_{j}^{\prime}, \mathbf{x}_{-j}\right)\right) \geq g_{i}\left(\tilde{q}\left(x_{j}, \mathbf{x}_{-j}\right)\right)+g_{i}\left(\tilde{q}\left(x_{j}^{\prime}, \mathbf{x}_{-j}\right)\right), \\
& g_{i}\left(\hat{q}\left(x_{j}, \mathbf{x}_{-j}^{\prime}\right)\right)+g_{i}\left(\hat{q}\left(x_{j}^{\prime}, \mathbf{x}_{-j}^{\prime}\right)\right) \geq g_{i}\left(\tilde{q}\left(x_{j}, \mathbf{x}_{-j}^{\prime}\right)\right)+g_{i}\left(\tilde{q}\left(x_{j}^{\prime}, \mathbf{x}_{-j}^{\prime}\right)\right),
\end{aligned}
$$

for each $i \in \mathcal{I}$ and, hence, $E_{\mathbf{x}}\left(\sum_{i} g_{i}(\hat{q}(\mathbf{x}))\right) \geq E_{\mathbf{x}}\left(\sum_{i} g_{i}(\tilde{q}(\mathbf{x}))\right)$. We iterate this procedure to obtain a sequence of allocations $q^{n} \in A$ and a decreasing numerical sequence $s^{n}=E_{\mathbf{x}}\left\|\mathbf{f}\left(q^{n}(\mathbf{x})\right)\right\|^{2}, n=1,2, \ldots$. If we find that $\check{f}_{j}\left(q_{j}^{n}\left(\cdot, \mathbf{x}_{-j}\right)\right)$ is non-decreasing for all $j$ and $\mathbf{x}_{-j}$ we set $q^{n+1} \equiv q^{n}$ and $s^{n+1} \equiv s^{n}$. Since $s^{n}$ is a weakly decreasing sequence bounded below by 0 it has a limit, which we denote as s. Since set $A$ is compact there also exists a convergent subsequence $q^{n}$ with a limit $q$ such that $q(\mathbf{x}) \in A$ for all $\mathbf{x} \in X$. Clearly, $s=E_{\mathbf{x}}\left(\| \mathbf{f}\left(q(\mathbf{x}) \|^{2}\right)\right.$ and $\check{f}_{j}\left(q_{j}\left(\cdot, \mathbf{x}_{-j}\right)\right)$ is non-decreasing for each $j$ 
and $\mathbf{x}_{-j}$. Since functions $g_{i}$ are continuous we also have $E_{\mathbf{x}}\left(\sum_{i} g_{i}(q(\mathbf{x}))\right) \geq E_{\mathbf{x}}\left(\sum_{i} g_{i}(\tilde{q}(\mathbf{x}))\right)$.

The result can then be further extended to continuous space with arbitrary distribution similar to Lemmas A1 and A2. We then use equation (6) to define payment rule $t$ delivering the same interim expected utilities. Finally, we have that the social surplus in the constructed allocation

$$
\begin{aligned}
E_{\mathbf{x}}\left(\sum_{i} v_{i}\left(q(\mathbf{x}), x_{i}\right)\right) & =E_{\mathbf{x}}\left(\sum_{i} f_{i}(q(\mathbf{x})) M_{i}\left(x_{i}\right)+m_{i}\left(x_{i}\right)+g_{i}(q(\mathbf{x}))\right) \\
& \geq E_{\mathbf{x}}\left(\sum_{i} f_{i}(\tilde{q}(\mathbf{x})) M_{i}\left(x_{i}\right)+m_{i}\left(x_{i}\right)+g_{i}(\tilde{q}(\mathbf{x}))\right)=E_{\mathbf{x}}\left(\sum_{i} v_{i}\left(\tilde{q}(\mathbf{x}), x_{i}\right)\right),
\end{aligned}
$$

where the inequality follows from the equal-marginal conditions in (4) and inequality (A.2). This establishes the claim of the theorem. The proof is analogues if instead condition (ii) is satisfied.

\section{Proofs of results stated in Section 5}

Proof of Corollaries 1 and 5. The statements follow from Theorem 1 .

Proof of Corollary 2, 3, and 4. The statements follow from Theorem 2 .

Proof of Corollary 6. Consider any BIC mechanism $(\tilde{q}, \tilde{t})$ and the equivalent DIC mechanism $(q, t)$, constructed in Theorem 1. Since we have $g_{i}(q)=0$ for each $i \in \mathcal{I}$ in the public good provision setting, we have that both mechanisms having the same ex ante expected utilities imply that both mechanism yield the same expected transfers, i.e. $E_{\mathbf{x}}\left(\sum_{i \in \mathcal{I}} t_{i}(\mathbf{x})\right)=E_{\mathbf{x}}\left(\sum_{i \in \mathcal{I}} \tilde{t}_{i}(\mathbf{x})\right)$.

To prove the claim of the corollary, we then need to show that the expected costs for DIC mechanism is lower than the expected costs for BIC mechanism, i.e. $E_{\mathbf{x}}(K(q(\mathbf{x}))) \leq E_{\mathbf{x}}(K(\tilde{q}(\mathbf{x})))$. This statement follows from applying the argument of the proof of Theorem 2 to function $-K$ instead of functions $g_{i}, i \in \mathcal{I}$. In particular, consider the sequence of allocation $q^{n}$ constructed in the algorithm of Theorem 1. Since function $K$ is non-decreasing and convex, the expected cost of allocations $q^{n}$ is non-increasing in $n$, i.e. $E_{\mathbf{x}}\left(K\left(q^{n+1}(\mathbf{x})\right)\right) \leq E_{\mathbf{x}}\left(K\left(q^{n}(\mathbf{x})\right)\right) \leq E_{\mathbf{x}}(K(\tilde{q}(\mathbf{x})))$. The continuity of $K$ then implies that the inequality holds in the limit. Finally, the result further extendeds to continuous type space with arbitrary distribution similar to Lemmas A1 and A2. 


\section{References}

Baliga, S. and Maskin, E. (2003): "Mechanism Design for the Environment," in K.G. Maler and J. Vincent, eds., Handbook of Environmental Economics, Vol. 1, Amsterdam: North-Holland, 305-324.

Borgers, T. (2015): An Introduction to the Theory of Mechanism Design, Oxford University Press.

Borgers, T. and Norman, P. (2009): "A Note on Budget Balance under Interim Participation Constraints: The Case of Independent Types," Economic Theory, 39, 477-489.

Duenyas, I., Hu, B., and Beil, R. B. (2013): "Simple Auctions for Supply Contracts," Management Science, 59, 2332-2342.

Gershkov, A., Goeree, J. K., Kushnir, A., Moldovanu, B., and Shi, X. (2013): "On the equivalence of Bayesian and dominant strategy implementation," Econometrica, 81, 197-220.

Goeree, J. K. and Kushnir, A. (2015): "A Geometric Approach to Mechanism Design," Working Paper, University of Technology Sydney and Carnegie Mellon University.

Hellwig, M. F. (2003): "Public-Good Provision with Many Participants," Review of Economic Studies, 70, 589-614.

Jehiel, P., Moldovanu, B. and Stachetti, E. (1996): "How (Not) to Sell Nuclear Weapons," American Economic Review, 86, 814-829.

Jehiel, P. and Moldovanu, B. (2006): "Allocative and Informational Externalities in Auctions and Related Mechanisms," in R. Blundell, W. Newey, and T. Persson, eds., The Proceedings of the 9th World Congress of the Econometric Society, Cambridge University Press, 2006.

Kushnir, A. (2015): "On Sufficiency of Dominant Strategy Implementation in Environments with Correlated Types," Economics Letters, 133, 4-6.

Laffont, J. J. and Martimort, D. (1997): "Collusion Under Asymmetric Information," Econometrica, $65,875-911$.

Ledyard, J. O. and Palfrey, T. R. (1999): "A Characterization of Interim Efficiency with Public Goods," Econometrica, 67, 435-448.

Mailath, G. J. and Postlewaite, A. (1990): "Asymmetric Information Bargaining Problems with Many Agents," Review of Economic Studies, 57, 351-367.

Manelli, A. M. and Vincent, D. R. (2010): "Bayesian and Dominant-Strategy Implementation in the Independent Private Values Model," Econometrica, 78, 1905-1938.

Martimort, D. and Sand-Zantman, W. (2013): "Solving the Global Warming Problem: Beyond Market, Simple Mechanism May Help!" Canadian Journal of Economics, 46, 361-378.

Martimort, D. and Sand-Zantman, W. (2015): "A Mechanism Design Approach to Climate-Change Agreements," Journal of the European Economic Association, forthcoming. 
Milgrom, P. R. (2004): Putting Auction Theory to Work, Cambridge University Press.

Milgrom, P. R. and Segal, I. (2002) "Envelope Theorems for Arbitrary Choice Sets," Econometrica, 70, 583-601.

Mookherjee, D. and Reichelstein, S. (1992): "Dominant Strategy Implementation of Bayesian Incentive Compatible Allocation Rules," Journal of Economic Theory, 56, 378-399.

Mookherjee, D. and Tsumagari, M. (2004): "The Organization of Supplier Network: Effects of Delegation and Intermediation," Econometrica, 72, 1179-1219.

Norman, P. (2004): "Efficient Mechanisms for Public Goods with Use Exclusions," Review of Economic Studies, 71, 1163-1188.

Royden, H. L. (1988): Real Analysis, New York: Macmillan Publishing Company.

Segal, I. (1999): "Contracting with Externalities," Quaterly Journal of Economics, 2, 337-388.

Severinov, S. (2008): "The Value of Information and Optimal Organization," RAND Journal of Economics, 39, 238-265. 